\title{
Echtzeitfähige Motorprozessmodelle für Schiffsmaschinen-Simulatoren
}

\author{
Georg Finger ${ }^{1^{*}}$, Karsten Wehner ${ }^{1}$, Egon Hassel $^{2}$, Steffen Loest ${ }^{1}$, \\ Michael Baldauf ${ }^{1}$ \\ 1Department of Maritime Studies ISSIMS Institute, University of Applied Sciences Wismar, Richard-Wagner-Str. 31,
18119 Rostock-Warnemünde, Germany; *georg.finger@hs-wismar.de
${ }^{2}$ Chair of Technical Thermodynamics, Rostock University, Albert-Einstein-Straße 2, 18059 Rostock, Germany
}

Abstract. Ein ökologisch sinnvoller und ökonomisch effizienter Schiffsbetrieb ist eine zwingende Grundanforderung der (nationalen und internationalen) Schifffahrt und kann durch vielfältige Maßnahmen erreicht werden. Viele dieser Maßnahmen sind technologische Ansätze, wie z.B. die Veränderung von Motorparametern oder Steuerungssystemen, Nutzung von alternativen Kraftstoffen, Anwendung von Abgasreinigungssystemen oder innermotorische Maßnahmen um Motorprozesse effizienter zu gestalten. Bei der Umsetzung dieser Maßnahmen wird oft außer Acht gelassen, dass immer noch Menschen an Bord sind, die für Betrieb und Wartung des Schiffes verantwortlich sind. Dieses Personal muss seine Aufgaben im Spannungsfeld von Umweltschutz und Effizienz unter allen auftretenden äußeren Bedingungen erfüllen. Die Ausbildung von technischen und nautischen Offizieren an Bord von seegehenden Schiffen beinhaltet neben der theoretischen Ausbildung an Land und der praktischen Seefahrtzeit auch Trainingseinheiten in Full-Mission Simulatoren. Diese Simulatoren, müssen auf die sich ändernden Ausbildungsanforderungen durch gezielte Verbesserung der integrierten Prozessmodelle weiterentwickelt werden.

In diesem Beitrag wird ein Ansatz zur echtzeitfähigen Integration von „Zwei-Zonen-Modellen“ zur Bestimmung von Stickoxiden im Abgas vorgestellt. Durch die zusätzliche Integration von Rußmodellen wird darüber hinaus auch die Darstellung von Rußemissionen z.B. im Hafen ermöglicht. Es wird demonstriert, wie durch den Einsatz von Assistenzsystemen der Verbrauch von Kraftstoff reduziert und eine Emissionsminderung im Hafen realisiert werden kann. Die Validierung der Ergebnisse erfolgt durch vergleichende Betrachtungen von realen Messdaten eines Prüfstandsmotor MAN $6 \mathrm{~L} / 2330$ mit den neu entwickelten und testweise integrierten Simulationsmodulen im Schiffsmaschinensimulator. Mit den FuE-Arbeiten wird ein Beitrag zum besseren Systemverständnis des nautischen und technischen Schiffsführungspersonals erbracht und die aktive Beeinflussung von Emissionen durch ökologisch sinnvolle und ökonomisch effiziente Steuereingriffe gefördert.

\section{Einleitung}

Technische und nautische Schiffsoffiziere unterliegen in ihren Ausbildungsanforderungen unterschiedlichen Standards. Die Mindeststandards werden in der International Convention on Standards of Training, Certification and Watchkeeping for Seafarers (nachfolgend STCW-Abkommen) geregelt. Dieses Abkommen legt fest, welchen Kenntnisstand Mannschaftsdienstgrade und Offiziere in Ihren jeweiligen Positionen vorweisen müssen. Neben rein theoretischem Wissen wird dabei auch praktische Erfahrung bei Anwendung bestimmter Verfahren und Methoden eingefordert. Eine Ausbildung findet dazu im Regelfall nicht nur an Bord von Schiffen statt, sondern in speziellen Simulatoren wie z.B. den am Bereich Seefahrt, Anlagentechnik und Logistik installierten Simulatoren für Schiffsführung (Ship Handling Simulator - SHS) und Schiffsmaschinenbetrieb (Ship Engine Simulator - SES). Beide Simulatoren sind vom DNV-GL als sogenannte Full-Mission Simulatoren zertifiziert und stellen bereits in Teilen eine detaillierte realistische Schiffsumgebung nach. So wird z.B. im SHS mit Hilfe von realen Konsolen in Verbindung mit einer $360^{\circ}$ - Sichtumgebung ein komplexes Seegebiet dargestellt und Trainees mit dem Verhalten eines Schiffes bei verschiedenen Umweltbedingungen vertraut gemacht [16]. Dabei wurde bisher der Fokus auf native Bewegungsmodelle gelegt. Eine Berücksichtigung des realen Verhaltens von Großmotoren findet in der nautischen Ausbildung bisher nur sehr oberflächlich statt. Bessere Möglichkeiten bietet ein Schiffsmaschinensimulator der alle an Bord von Schiffen vorhanden Versorgungs- und Antriebssysteme nachbilden kann. Allerdings liegt der Schwerpunkt auf dem reinen Betrieb von Großmotoren. Die dabei entstehenden Emissionen ließen sich bisher nicht zufriedenstellend darstellen.

Dieser Artikel befasst sich mit der Entwicklung von Prozessmodellen zur Emissionsbildung und von Kraftstoffverbräuchen sowie deren Anwendung in Simulatoren und Assistenzsystemen. 


\section{Einfluss von Assistenzsystemen auf den Kraftstoffverbrauch}

Im Rahmen des vom Bundesministerium für Wirtschaft und Energie geförderten Verbundprojektes MEmBRan zur Modellierung von Emissionen und Brennstoffverbräuchen wurden experimentelle und Feldstudien durchgeführt, welche eine Betrachtung des reinen Energiebedarfs am Propeller beim Manövrieren mit unterschiedlichen Assistenzsystemen verfolgte. Zunächst wurden dazu Hafenanläufe von realen Schiffen analysiert um eine Basis für den unterschiedlichen Umgang von nautischen Offizieren mit Ihren Schiffen darzustellen. Figure 1 zeigt dabei die Bandbreite der sich aus unterschiedlichen Manöverstrategien ergebenden Bahnverläufe beim Anlegen. Dabei stellt die rote Linie die Wegpunktliste der von der Schiffsführung geplanten Route dar, während die schwarzen Linien die tatsächlich zurückgelegten Strecken sind.

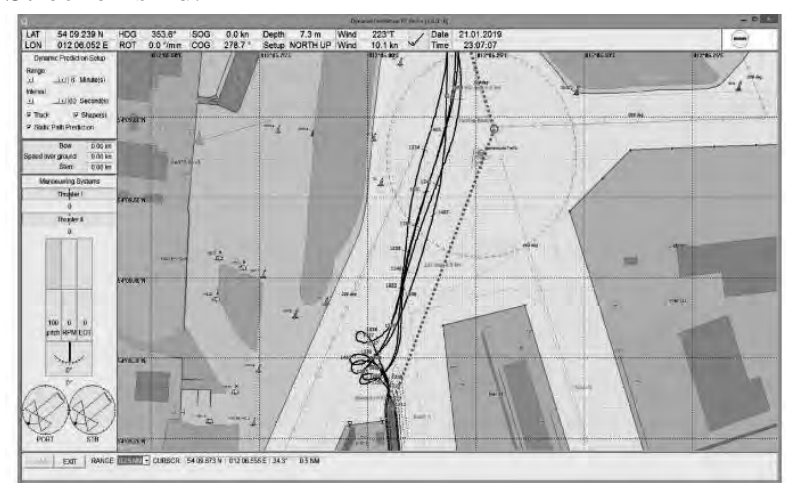

Figure 1: Hafenanlaufstrategien verschiedener Offiziere

Hierbei variierte der Leistungsbedarf an den Propellern im Manöverbetrieb um bis zu 20\%[1]. Da in der realen Umgebung verschiedene Umwelteinflüsse eine Rolle spielen und eine Vergleichbarkeit dabei nur schwer erzielt werden kann, wurden weitere Versuche im Schiffsführungssimulator unter kontrollierbaren Bedingungen durchgeführt. Zur Vergleichbarkeit wurden drei verschiedene Szenarien entwickelt und in einem FullMission-Simulator, in denen die Probanden, erfahrene Nautiker und Kapitäne, mit dem Schiff anlegen sollten, eine Durchfahrt durch eine fiktive Insellandschaft vollführen oder bis zu einem Ankerplatz manövrieren mussten. Dabei hat jede Testperson jedes Szenario nur einmal durchgeführt um etwaige Lernprozesse auszuschließen. Ein Szenario wurde dabei „klassisch“ - das heißt ohne die Zuhilfenahme von Assistenzsystemen durchgeführt.
Ein Szenario erfolgte unter Zuhilfenahme eines Manöverplanungswerkzeuges. Bei diesem Werkzeug wird im Vorfeld der Übung festgelegt an welchem geographischen Punkt, welcher Steuereingriff (Manöver) erfolgt. Im Gegensatz zu einer reinen Wegpunktliste, bei denen jeweils nur die anzufahrenden geografischen Positionen geplant sind, enthält der Manöverplan, daher exakte Anweisung zum Einsatz der einzelnen Kontrollorgange, wie beispielsweise Ruderlage oder zu ordernde Propellerdrehzahl. Für dieses, am Institut für Innovative Schiffssimulation des Bereiches Seefahrt, Anlagentechnik und Logistik entwickelte, Werkzeug ist daher eine exakte Modellierung des Bewegungsverhaltens erforderlich

Figure 2 zeigt einen Auszug aus einem Manöverplan mit verschiedenen Manöverpunkten. Somit wird aus einem rein mentalen Modell des Nautikers ein vorhersagbares und reproduzierbares Vorgehen geschaffen.

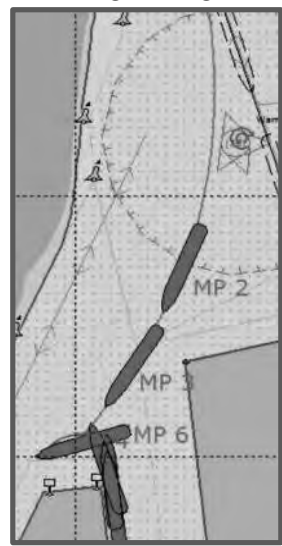

Figure 2: Auszug aus Manöverplan

Als weiteres Assistenzsystem wurde den Probanden eine Fast Time Simulation zur Verfügung gestellt, die den Probanden die Schiffsbewegung für einen Vorab definierten Zeitraum während der Übung grafisch darstellt. Somit hatten die Testpersonen während der Durchführung die Möglichkeit, ihre Handlungen zu validieren. Figure 3 stellt die durchschnittliche Leistungsaufnahme des Propellers dar. In den Versuchen zeigte sich, dass bei den mittels Assistenzsystemen durchgeführten Anlegemanövern die benötigte Propellerleistung signifikant geringer war Mit Steigerung des Assistenzgrades sinkt der Leistungsbedarf um knapp 30\%. Dieser Effekt wird besonders in küstennahen Gebieten und beim Manövrieren im Hafen deutlich. Bei langen Überfahrten verschwindet der Effekt. Bei einem Schiff, das sehr viele Manöver in kurzer Zeit ausführen muss, wie beispielsweise Fähren im Kurzstreckenverkehr, ist der Effekt sehr deutlich messbar. 


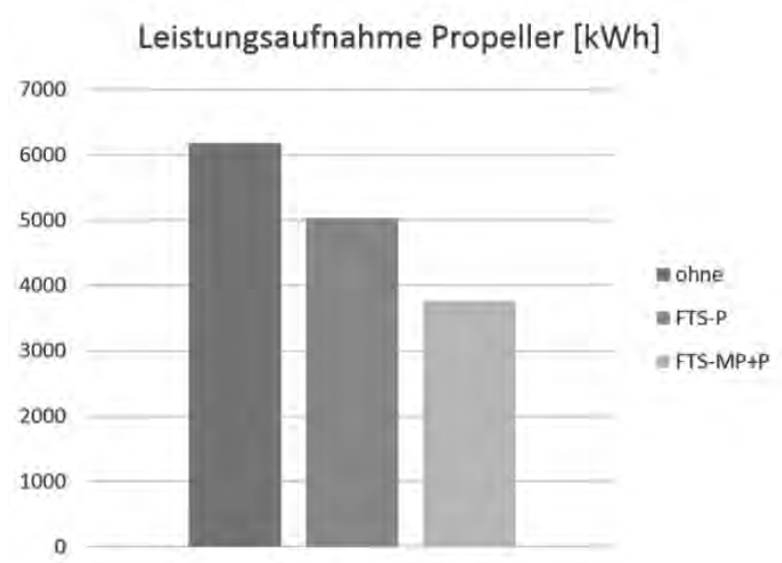

Figure 3: Durchschnittsergebnis aus einem Szenario

Zurückzuführen lässt sich diese Ersparnis auf zwei Parameter. Zunächst werden die Anzahl und Intensität der Steuereingriffe (Manöver) minimiert. Beispielsweise kann eine übermäßige Beschleunigung, die im weiteren Verlauf wieder abgefangen werden muss, vorausschauend vermieden oder minimiert werden. Des Weiteren sinkt der Einfluss von Ruderkommandos. Jedes Ruderkommando hat einen Einfluss auf die Anströmung von Propeller und Schiffskörper und hat somit eine Wiederstandsänderung zur Folge. Übermäßige Quergeschwindigkeiten, führen somit immer zu einem zusätzlichen Leistungsbedarf des Propellers. Figure 4 zeigt die Gegenüberstellung von Ruderwinkeln eines Szenarios ohne Assistenzsystem (oben) und mit Prädiktion und Planung (unten). Man kann erkennen, dass die Anzahl an Korrekturen stark abnimmt, somit große Ruderlagen größtenteils bis zum Erreichen des nächsten Manöverpunktes konstant bleiben.

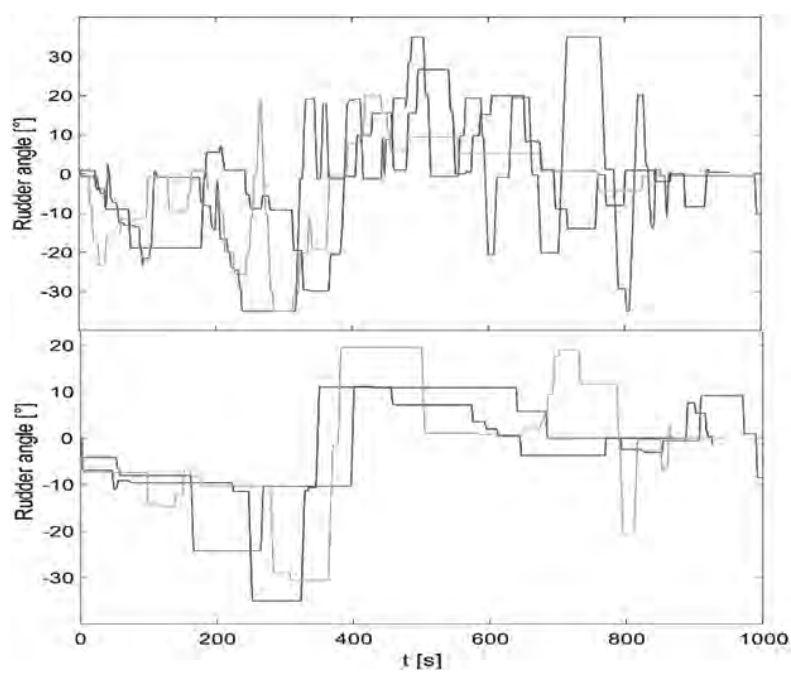

Figure 4: Ruderwinkel aus einem Szenario
Unzureichend an dieser Darstellung ist bisher, dass lediglich die am Propeller abgeforderte Leistung auf Basis des Bewegungsmodells quantifiziert wird. Technisch findet sowohl in der Prädiktion, als auch in der Simulation des Schiffsführungssimulators keine reale Simulation des Motorprozesses statt. Das führt dazu das somit keine Aussagen über den realen Kraftstoffverbrauch und Emissionen wie beispielsweise Stickoxide oder Ruß stattfinden kann, da der Betriebspunkt des Motors nicht bekannt ist. Somit wurde eine Kopplung des Schiffsführungssimulators mit dem Schiffsmaschinensimulator inklusive Integration von Brennraum- und Emissionsmodellen angestrebt.

\section{Motormodell}

Das Motormodell besteht aus verschiedenen Komponenten die nachfolgend näher betrachtet werden. Die Komponenten lassen sich in Regler, Turboladermodell und Zylinderinnenprozess aufteilen. Im Innenprozess findet dabei der Energieumsatz statt auf dessen Basis die Emissionsmodelle aufbauen.

\subsection{Grundlagen des Zylinderinnenprozesses}

Der Zylinderinnenprozess basiert auf dem ersten Hauptsatz der Thermodynamik für ein offenes System (Fehler! Verweisquelle konnte nicht gefunden werden.).

$$
\frac{d U}{d t}=-p \frac{d V}{d t}+\dot{Q}_{B}+\dot{Q}_{W}+\dot{H}_{B B}+\dot{H}_{\text {in }}-\dot{H}_{o u t}
$$

Darin beschreibt der Term $-p \frac{d V}{d t}$ die Volumenänderungsarbeit des Gases die letztlich an den Kolben abgeben wird und letztendlich über die Welle die Arbeit unter Abzug von Reibungsverlusten am Propeller verrichtet. $\dot{Q}_{B}$ ist die im Brennstoff chemisch gebundene Energie die nach Einspritzung und Verdampfung umgesetzt wird. Der Wandwärmestrom $\dot{Q}_{W}$ beinhaltet die Wärme die Vom Brennraum an die Wände von Laufbuchse, Kolben und Zylinderdeckel abgeben wird und über das Kühlwasser des Motors abgeführt wird. Die Enthalpieströme $\dot{H}_{\text {in }} \& \dot{H}_{\text {out }}$ enthält die Energie die mit dem Gas über Einund Auslassventile aus dem Brennraum transportiert wird. Der Enthalpiestrom $\dot{H}_{B B}$ berücksichtigt dabei die 
Verluste über Undichtigkeiten der Reibpaarung von Kolbenring und Laufbuchse. Die Berechnung der spezifischen inneren Energie u erfolgt dabei nach dem Ansatz von Justi [2](2)

$$
\begin{gathered}
u(T, \lambda)=0,1445\left[1356,+\left(489,6+\frac{46,4}{\lambda^{0,93}}\right) *\right. \\
*\left(T-T_{B e z}\right) 10^{-2}+ \\
+\left(7,768+\frac{3,36}{\lambda^{0,8}}\right)\left(T-T_{B e z}\right)^{2} 10^{-4}- \\
\left.-\left(0,0975+\frac{0,0485}{\lambda^{0,75}}\right)\left(T-T_{B e z}\right)^{3} 10^{-6}\right]
\end{gathered}
$$

Basierend auf den partiellen Ableitungen aus (1) und (2) unter Berücksichtigung des Verbrennungsluftverhältnisses $\lambda$ lässt sich zu jedem Zeitpunkt bzw. zu jedem Grad Kurbelwinkel $\varphi$ die thermodynamische Durchschnittstemperatur T bestimmen. Aus der Temperatur lässt sich über die allgemeine Gasgleichung der Druck im Brennraum bestimmen und über die Brennraumgeometrie die Kraft auf den Kolben, welche letztendlich in das Drehmoment der Welle übergeht. Da zur Bestimmung des Energieumsatzes das Verbrennungsluftverhältnis benötigt wird, ist die genaue Kenntnis des Ladeluftdrucks nach Turboladerkompressor und Ladeluftkühler sowie des Abgasgegendruckes vor der Turboladerturbine unerlässlich. Sind die Drücke vor und nach Zylinder bekannt, so lässt sich der Massenstrom über Ein- und Auslassventil durch die dynamischen geometrischen Größen durch die Modellierung einer Drosselstelle (3) wiedergeben.

$$
\dot{m}=A_{1} \sqrt{\rho_{1} * p_{1}} * \sqrt{\frac{2 \kappa}{\kappa-1}\left[\left(\frac{p_{2}}{p_{1}}\right)^{\frac{2}{\kappa}}-\left(\frac{p_{2}}{p_{1}}\right)^{\frac{\kappa+1}{\kappa}}\right]}
$$

Über den Massenstrom und die innere Energie lassen sich somit die Enthalpieströme darstellen. Unter Berücksichtigung der Kraftstoffzuführung aus Kapitel 2.2 lässt sich somit der Brennraumdruck über ein Arbeitsspiel numerisch berechnen. In Figure 5 sind die Berechnungsergebnisse dargestellt die aus diesen Ansätzen statisch hervorgehen. Die schwarze Linie ist dabei eine Kurve die zwischen 2.5 und 3 bar verläuft und den Ladungswechsel darstellt.

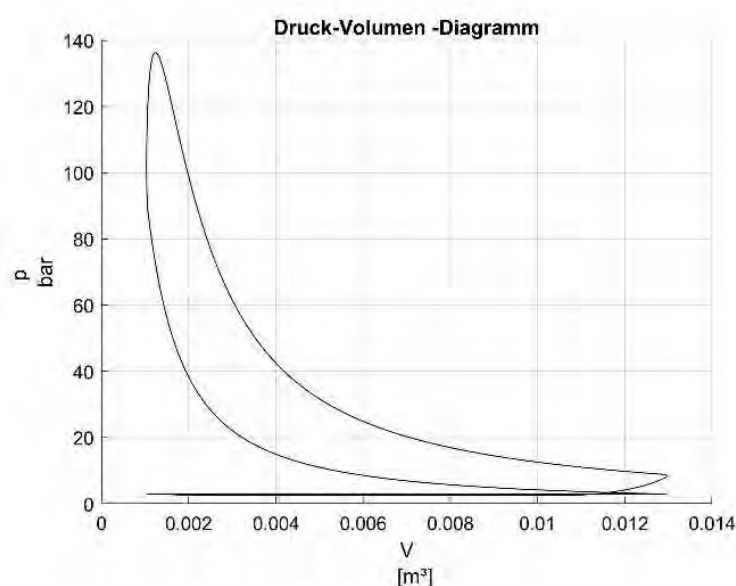

Figure 5: Druck-Volumen-Diagramm

\subsection{Kraftstoffzufuhr und Umsetzung}

Die Kraftstoffzufuhr zum Motor eines Schiffes wird äußerlich über zwei Komponenten geregelt. Im Vordergrund steht zunächst die Drehzahlanforderung der nautischen Schiffsführung über den Maschinentelegraphen. Dieser Sollwert wird in der Regel über Hochfahrprogramme gefiltert um eine Überlast des Motors zu minimieren. Für jedes Schiff werden diese Programme neu abgestimmt, aufgrund der Tatsache, dass sich ein Schiffseigner für eine beliebige - technisch mögliche - Kombination von Rumpf, Propeller und Maschine entscheiden kann. Der Regler am Motor ist ein konventioneller PIDRegler, der die benötigte Kraftstoffmenge auf den derzeitigen Sollwert einregelt. Programmtechnisch lassen sich beide Varianten zusammenfassen. Ein Motor mit klassischer Einspritzung über Pumpe-Düse Verfahren ist dabei im Kraftstoffmassenstrom über die Drehzahl begrenzt. Figure 6 zeigt exemplarisch die Beschleunigung des Versuchsmotors auf Nennleistung.

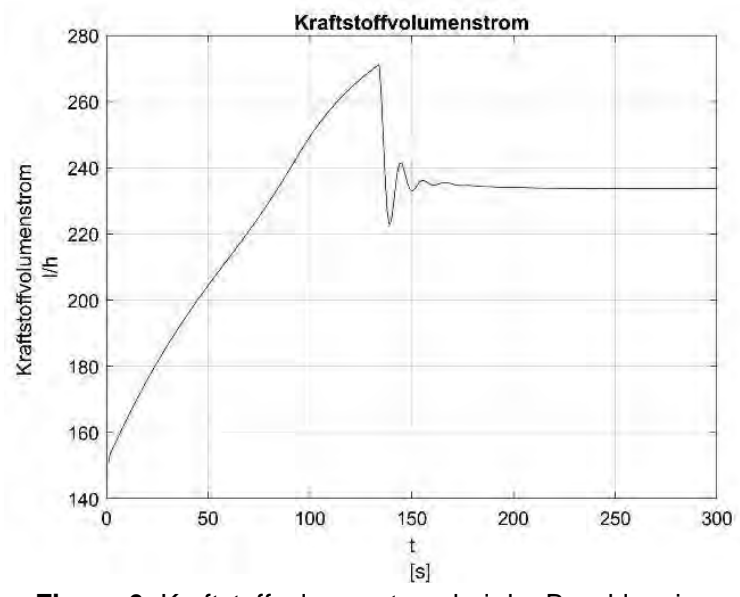

Figure 6: Kraftstoffvolumenstrom bei der Beschleunigung 
Leicht ersichtlich ist in diesem Beispiel, dass der Kraftstoffverbrauch nach dem Überschwingen noch absinkt. Dies ist auf den verbesserten Umsatz in Folge höherer Ladeluftdrucke zurückzuführen. Das hier dargestellte Simulationsergebnis basiert auf einer „stand-alone“ Variante die etwaige Beschleunigungsmomente aus den Bewegungsgleichungen aus Abschnitt1 noch nicht ausreichend berücksichtigt. Da eine reine Simulation der Kraftstoffzufuhr über das Füllungsgestänge den Eintrag und die Umsetzung im Zylinderinnenprozess nicht wiederspiegelt, wurde der Ansatz nach Vibe [3] verwendet, um den Kraftstoffmassenstrom in den Energieumsatz $\dot{Q}_{B}$ aus (1) zu übertragen. Dabei sind Anpassungen nach Sitkei[4] und Woschni et al[5] dynamisch berücksichtigt, sodass sich der Brennverlauf $\dot{Q}_{B}(\varphi)$ dynamisch an äußere Umstände anpassen lässt.

\subsection{Turboladermodell}

Um die Drücke vor und nach Zylinder zu berechnen sind exakte Kenntnisse über das Betriebsverhalten des Turboladers und der jeweiligen Sammelrohre notwendig. Aufgrund der Tatsache, dass der Turbolader seine Antriebsenergie zur Verdichtung von Ladeluft aus der Abgasenergie nach dem Zylinder bezieht, ist es ein rückgekoppeltes System. Das System kann in der numerischen Berechnung, insbesondere bei sehr großen Schrittweiten, schnell instabil werden, da eine stationäre Simulation immer auf dem Gleichgewicht von Antriebsmoment der Turbine $M_{T}$ und Arbeitsmoment des Kompressors $M_{C}$. Dies hat in der Simulation eine ungewollte Wechselwirkung zwischen Ladeluftdruck und Drehzahl zur Folge. Die Berechnung beruht dabei auf den technisch validierten Modellen der Turboladerhauptgleichung [6]. Dieses Modell ist skalierbar und ebenfalls auf unterschiedliche Turbolader/Motorkonstellationen anpassbar. Die Ergebnisse der Parametrierung des Modells an den Prüfstandsmotor des Bereichs Seefahrt, Anlagentechnik und Logistik MAN 6L23/30 sind in Figure 7 ersichtlich. Hier ist ein dynamischer Lastsprung zwischen Messung („Eng“ - in Abhängigkeit konventioneller Schiffsautomatisierungstechnik in 0.1 bar Schritten) und Simulation („Sim“) dargestellt. Der zeitliche Verlauf hat einen hohen Einfluss auf die Emissionen, da die Luftmasse im Zylinder den Verbrennungsvorgang maßgeblich bestimmt.

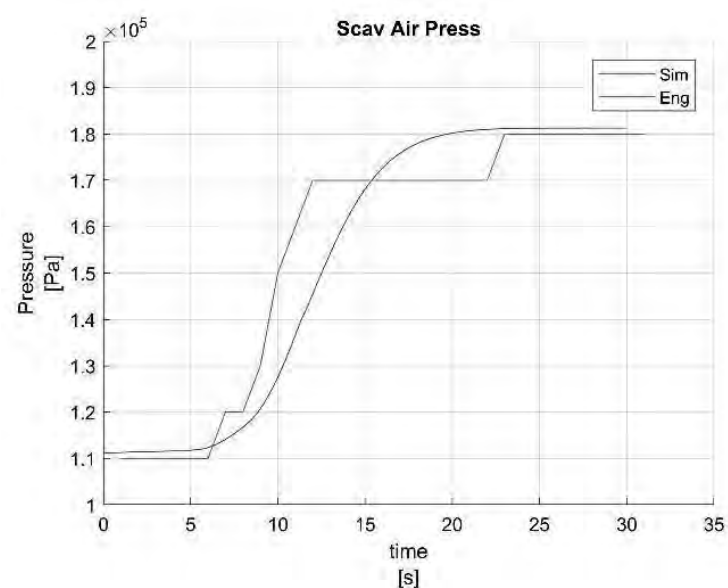

Figure 7: Ladeluftdruck Simulation und Messung

\subsection{Emissionsmodell}

Die Berechnung der Emissionen sind abhängig von den vorausgegangen Berechnungen und basieren jeweils auf Druck- und Temperaturwerten im Brennraum. Daher sind die zuvor beschriebenen Berechnungen unabdingbare Basis für die Modellierung von Emissionen.

\section{Stickoxid-Modell}

Die Modellierung von Stickoxiden basiert auf den in Kapitel 2.1 beschriebenen Berechnungen. Zur Berechnung der Volumenänderungsarbeit ist hier die thermodynamische Durchschnittstemperatur ausreichend. Thermische Stickoxide entstehen aus dem in der Verbrennungsluft enthaltenen Stickstoff. Hohe Temperaturen in der Flammenfront sind daher erforderlich, um die Dreifachbindung des Luftstickstoffs aufzubrechen. Daher wird nach Heider[7] die thermodynamische Durchschnittstemperatur in zwei Zonen aufgeteilt. Zone eins beinhaltet das unverbrannte Material und Zone zwei ist die bereits verbrannte Zone. Die Aufteilung erfolgt dabei nach der Funktion $B(\varphi)$ (4) unter Berücksichtigung des motorspezifischen Faktors $A^{*}$

$$
T_{1}(\varphi)-T_{2}(\varphi)=B(\varphi) A^{*}
$$

Die Berechnung erfolgt dabei basierend auf dem Schleppdruck $p_{0}$ eines Motors ohne Verbrennung und dem Integral des Druckverlaufs $\int_{\varphi B C}^{\varphi E x V o}$ von Brennbeginn (BC) und Öffnung des Auslassventils (ExVo) mit der Funktion (5) 


$$
B(\varphi)=1-\frac{\int_{\varphi B C}^{\varphi}\left[p(\varphi)-p_{0}(\varphi)\right] m_{1} d \varphi}{\int_{\varphi B C}^{\varphi E x V o}\left[p(\varphi)-p_{0}(\varphi)\right] m_{1} d \varphi}
$$

Durch die Temperatur in der Flammenfront lässt sich mit Hilfe des Zeldovich-Mechanismus[8](6) die Konzentrationsänderung von Stickstoffmonooxid bestimmen.

$$
\begin{gathered}
\frac{d[\mathrm{NO}]}{d t}=k_{1, r}[\mathrm{O}]\left[\mathrm{N}_{2}\right]+k_{2, r}[\mathrm{~N}]\left[\mathrm{O}_{2}\right]+k_{3, r}[\mathrm{~N}][\mathrm{OH}] \\
\quad-k_{1, l}[\mathrm{NO}][\mathrm{N}]-k_{2, l}[\mathrm{NO}][\mathrm{O}]-k_{3, l}[\mathrm{NO}][\mathrm{H}]
\end{gathered}
$$

Der Geschwindigkeitsbestimmende Faktor ist in Folge der hohen Aktivierungsenergie $k_{1, r}$ und kann der Literatur entnommen werden [9][10][11][12]. Eine Bestimmung der exakten Temperaturen nach diesen Faktoren ist dabei unerlässlich. Eine Abweichung um vier Kelvin im Bereich von 1600K führt bereits zu einer um bis zu 10\% fehlerhaften Berechnung der Stickstoffmonoxidbildung.

\section{Ruß-Modell}

Die Modellierung von Ruß in echtzeitfähigen Verfahren ist derzeit nur beschränkt möglich. Genaue Verfahren basieren auf CFD-Rechnungen und sind weit verbreitet, aber auch rechenintensiv. Als weit verbreitetes Modell dient das Modell von Nishida und Hiroyasu [13]. In diesem Modell werden Rußbildung (7) und Oxidation (8) durch empirische Gleichung beschrieben und die Bestimmung der Gesamtrußentstehung erfolgt durch Differenzbildung der beiden Größen.

$$
\begin{gathered}
\frac{d m_{P, B}}{d t}=A_{B} * m_{B, g} p^{0,5} e^{-\frac{6313}{T}} \\
\frac{d m_{P, O x}}{d t}=A_{O x} * m_{p} x_{O_{2}} * p^{1,8} e^{-\frac{7070}{T}}
\end{gathered}
$$

Dieser Ansatz basiert auf der gasförmigen Brennstoffmasse $m_{B, g}$ und dem Sauerstoffstoffmengenanteil $x_{O_{2}}$ sowie den Druck- und Temperaturwerten im Zylinder. Die Berechnung erfolgt als Paketmodell im Einspritzstrahl. Ebenfalls erfolgt eine Anpassung durch die motorspezifischen Faktoren $A_{B}$ und $A_{O x}$. Dieses Modell wurde nun adaptiert und in einem Einzonenmodell getestet und in den SES statisch implementiert. Das Rechenergebnis der Partikelbeladung in Milligramm pro Kubikmeter ist für die Trainees allerdings sehr unhandlich. Daher erfolgt eine iterative Umrechnung in die Filterschwärzungszahl nach AVL [14] (9).

$$
P B=\frac{1}{0.405} * 4,95 * F S N e^{0,38 F S N}
$$

Diese kann wiederum in eine Opazität umgerechnet werden. Somit lässt sich im Simulator eine schwarze Rußfahne bei ungünstigen Lastzuständen darstellen, um eine visuelle Rückkopplung an den Trainee zu geben. Figure 8 stellt dabei die Rußbildung an verschiedenen stationären Lastpunkten gegenüber.

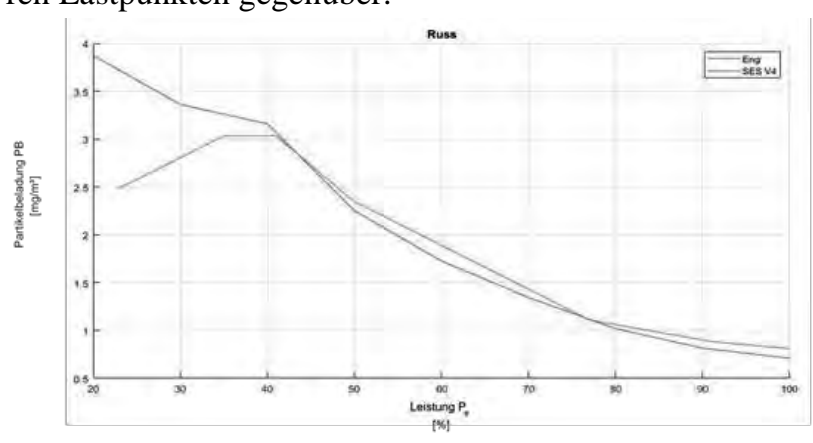

Figure 8: Rußbildung stationärer Lastpunkte

In Figure 9 ist das Ergebnis eines Lastsprunges auf einer Propellerkurve dargestellt. Die Abweichungen zwischen Simulation und Prüfstand sind auf die Tatsache zurückzuführen, dass das Ergebnis der Rußberechnung nach Zylinder erfolgt, während die Messung am Prüfstand nach dem Turbolader erfolgt und dementsprechend Mischungsverhätnisse und Trägheiten nicht vollständig berücksichtigt sind.

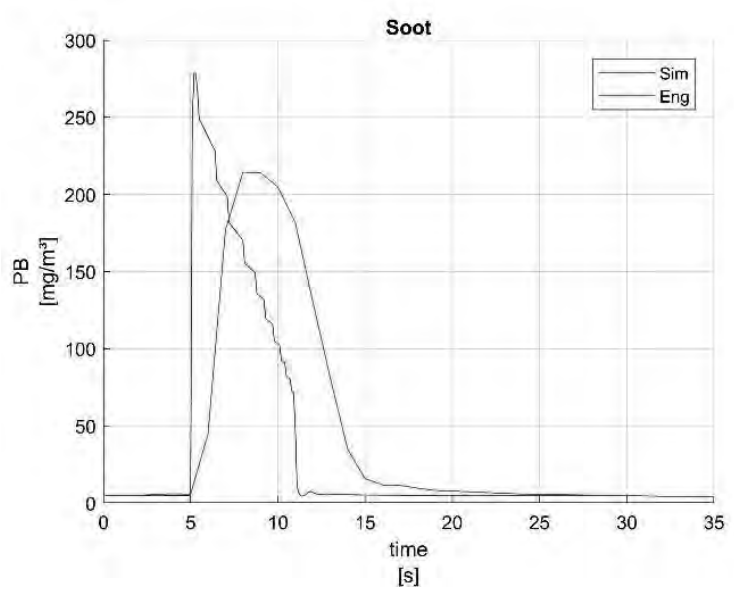

Figure 9: Rußbildung währende eines Lastsprunges

\subsection{Zusammenfassung und Ausblick}

Die in diesem Beitrag dargestellten Methoden zur Modellbildung von Motorprozessen ermöglichen eine 
komplexe und umfassendere Schiffssimulation. Die Anwendung auf vorhergehende Untersuchung zeigt, dass der Kraftstoffbedarf quantifizierbar wird und somit auch in die nautische Manöverplanung einfließen kann. Bisher erfolgte die Umsetzung der Motorprozesse in 3 Phasen. Sowohl der C-Code des Schiffsmaschinensimulators, als auch die C++ Umgebung zur Entwicklung des Bewegungsverhaltens des Schiffes sind echtzeitfähig. Problematisch wird die Berechnung bei sehr hohen Drehzahlen über 4000 rpm. Da dort nicht mehr sämtliche Umdrehungen gerechnet werden können. Ebenfalls erweist sich eine Einbindung in die Fast-Time-Simulation zur Prädiktion im operativen Betrieb als schwierig. Da dort Vorausberechnung bis 24min erfolgen sollen, ist der Rechenaufwand durch das Motormodell immens. Abhilfe schafft an dieser Stelle die Einbindung künstlicher neuronaler Netze. Diese können mit den Ergebnissen des Simulationstools angelernt werden und beschleunigen die Berechnung um ein Vielfaches. Erste Vorarbeiten sind von Schaub et al.[15] bereits durchgeführt worden. Des Weiteren müssen weitere Modelle eingepflegt bzw. angepasst werden, um nicht nur der Vielfalt an Motoren gerecht zu werden, sondern auch, um nicht-konventionelle Motoren wie Common-Rail oder Dual-Fuel Motoren zukünftig darzustellen.

\section{Danksagung}

Der in diesem Beitrag beschriebene Ansatz wurde im Rahmen des vom Bundesministeriums für Wirtschaft und Energie geförderten FuE-Projektes MEmBRan entwickelt und getestet. An diesem Projekt waren beteiligt: MARSIG GmbH Rostock, Hochschule Wismar (Bereich Seefahrt, Anlagentechnik und Logistik), Universität Rostock (Lehrstuhl für Technische Thermodynamik), Rheinmetall Electronics Bremen (Abteilung Maritimeund Prozesssimulation) und assoziierte Partner.

\section{Nomenklatur}

SHS - Schiffsführungssimulator

SES - Schiffsmaschinensimulator

U - innere Energie [J]

$\mathrm{H} \quad$ - Enthalpie [J]

$\mathrm{V} \quad$ - Volumen $\left[\mathrm{m}^{3}\right]$

$\mathrm{p} \quad$ - Druck [Pa]

$\dot{Q}_{B} \quad$ - Energiefreisetzung aus Brennstoff [kJ/s]

$\dot{Q}_{W} \quad$-Wandwärmestrom [kW]

$\lambda \quad$-Verbrennunsluftverhältnis [-]

$\varphi \quad$ - Kurbelwinkel [ ${ }^{\circ}$ ]

$\mathrm{T} \quad$ - Temperatur im Brennraum [K]

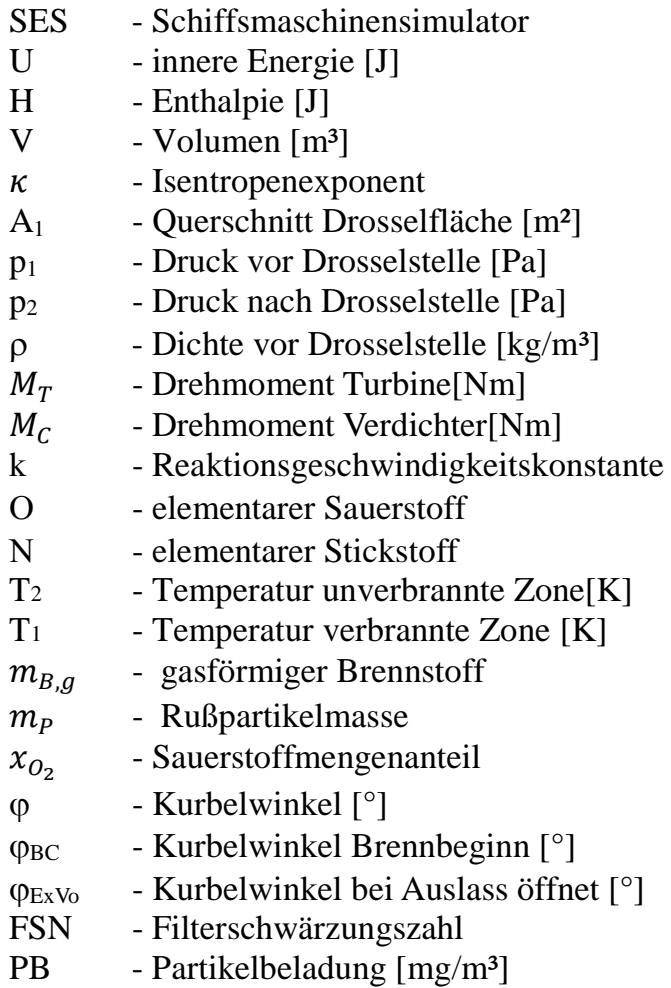

\section{References}

[1] Finger, G., Schaub, M., Dahms, F., Hassel, E., Riebe, T., Milbradt, G. u. Wehner, K.: On-board Support System for the eco-friendly ship operation in coastal and port areas. In: OCEANS 2019 (Hrsg.): Proceedings OCEANS 2019. Marseille 2019

[2] Justi, E.: Spezifische Wärme, Enthalpie, Entropie und Dissoziation technischer Gase , Springer Verlag (1938)

[3] Vibe, I.: Brennverlauf und Kreisprozess von Verbrennungsmotoeren, VEB Verlag Technik, Berlin (1970)

[4] Sitkei, G.: Über den dieselmotorischen Zündverzug. Motortechnische Zeitschrift MTZ 26 (1963)

[5] Woschni, G., Anisits, F.:Eine Methode zur Vorausberechnung der Änderung des Brennverlaufs mittelschnellaufender Dieselmotoren bei geänderten Betriebsbedingungen. Motortechnische Zeitschrift MTZ 34, 106 ff., Franckh-Kosmos Verlags- GmbH, Stuttgart (1973)

[6] Merker, P.: Title Grundlagen Verbrennungsmotoren. p 228

[7] Heider, G.: Rechenmodell zur Vorausberechnung der NO-Emissionen von Dieselmotoren, Dissertation, TU München (1996)

[8] Zeldovich,Y.B.: The Oxidation of Nitrogen in Combustion and Exlosion. Acta Physicimica ,USSR 21, pp. 577628 (1946)

[9] GRI-MECH 3.0: www.me.berkeley.edu/gri_mech (2000)

[10] Baulch, Evaluated kinetic data for combustion modelling. Supplement I, Journal of Physical and Chemical 
Reference Data 23, pp. 847 (1994)

[11] Heywood, J.B.: Internal Combustion Engine Fundamentals. McGraw-Hill (1988)

[12] Pattas, K.: Stickoxidbildung in der ottomotorischen Verbrennung, MTZ 34, pp. 397-404 (1973)

[13] Nishida, K., Hiroyasu, H. : Simplified Three-Dimensional Modeling of Mixture Formation and Combustion in a DI Diesel Engine, SAE Paper, 890269 (1989):

[14] AVL, Smoke value measurement with the filter-paper-method (2005)

[15] Schaub M, Data-Based Prediction Of Soot Emissions For Transient Engine Operation. IAPGOS 9(4):10-13. doi:10.35784/IAPGOS.29 (2019)

[16] Baldauf, M, Schröder-Hinrichs, JU, Kataria, A, Benedict $\mathrm{K}$, Tuschling, G (2016) Multidimensional simulation in team training for safety and security in maritime transportation, Journal of Transportation Safety \& Security, 8:3, 197-213, DOI: 10.1080/19439962.2014.996932 